\title{
Probing Chirality of Top-Higgs FCNC Couplings at Linear Colliders
}

\author{
Blaženka Melić * \\ Rudjer Bošković Institute, Division of Theoretical Physics, Bijenička 54, HR-10000 Zagreb, \\ Croatia \\ E-mail: meliceirb.hr \\ Monalisa Patra \\ Rudjer Bošković Institute, Division of Theoretical Physics, Bijenička 54, HR-10000 Zagreb, \\ Croatia \\ E-mail: Monalisa.Patra@irb.hr
}

\begin{abstract}
We study the nature of the top-Higgs flavor changing neutral current (FCNC) couplings in the $t \bar{t}$ production at polarized linear colliders. We show how the polarized linear colliders can be used to determine the chirality of the FCNC coupling by using the angular distributions of the top decay products and by examining relevant asymmetries along with the top spin polarizations and correlations. We obtain a limit on the couplings and find the $3 \sigma$ upper bound on $\operatorname{BR}(t \rightarrow q H)<$ $8.84 \times 10^{-4}$ at $\sqrt{s}=500 \mathrm{GeV}$ and $\mathscr{L}=500 \mathrm{fb}^{-1}$.
\end{abstract}

EPS-HEP 2017, European Physical Society conference on High Energy Physics 5-12 July 2017

Venice, Italy

${ }^{*}$ Speaker. 


\section{Introduction}

The FCNC transitions mediated by the Higgs boson are absent at tree level in the standard model (SM). The SM branching ratio of $t \rightarrow c H$ is loop induced and of the order of $\operatorname{BR}(t \rightarrow$ $c H)_{S M} \approx 10^{-15}$, which is many orders of magnitude smaller than the value to be measured at the $14 \mathrm{TeV}$ LHC. The absence of Higgs mediated tree level FCNC transitions within the SM is due to the presence of only one Higgs doublet. However these FCNC transitions can be large in the presence of additional scalar doublets or through the extra contribution of new particles in the loop diagrams as predicted in many extensions of the SM. The LHC has a dedicated program measuring the various properties of the Higgs boson, therefore an affirmative observation of the process $t \rightarrow q H$, differing from the SM rate, will be a conclusive indication of new physics beyond the SM. The most general FCNC $t q H$ Lagrangian considered is of the form

$$
\begin{aligned}
\mathscr{L}^{t q H} & =g_{t u} \bar{t}_{R} u_{L} H+g_{u t} \bar{u}_{R} t_{L} H+g_{t c} \bar{t}_{R} c_{L} H+g_{c t} \bar{c}_{R} t_{L} H+h . c \\
& =\bar{t}\left(g_{q t} P_{R}+g_{t q}^{*} P_{L}\right) q H+\bar{q}\left(g_{t q} P_{R}+g_{q t}^{*} P_{L}\right) t H .
\end{aligned}
$$

The total decay width of the top in the presence of these FCNC couplings can be then written as

$$
\Gamma_{t}=\Gamma_{t}^{S M}+\Gamma_{t \rightarrow q_{H}} \approx \Gamma_{t}^{S M}+0.155\left(\left|g_{t q}\right|^{2}+\left|g_{q t}\right|^{2}\right),
$$

and from the experimentally observed $\Gamma_{t}$ an upper bound on $\sqrt{\left|g_{t q}\right|^{2}+\left|g_{q t}\right|^{2}}$ can be obtained. It was shown in Ref. [1], that studying the single top + Higgs production in $p p \rightarrow\left(t \rightarrow W^{+} b\right) H$ at the LHC, will lead to the disentangling of the $t u H$ and $t c H$ couplings. Here we show that at linear colliders it is possible to distinguish the chirality of those couplings [2].

\section{Analysis of the $t q H$ final state at the polarized $e^{-} e^{+}$linear collider}

We consider the following process in the context of the $e^{-} e^{+}$collider,

$$
\begin{aligned}
e^{-}\left(p_{1}\right)+e^{+}\left(p_{2}\right) \rightarrow & t\left(q_{1}\right)+\bar{t}\left(q_{2}\right), \\
& t\left(q_{1}\right) \rightarrow q\left(p_{q}\right)+H, \quad \bar{t}\left(q_{2}\right) \rightarrow \bar{b}\left(p_{b}\right)+l^{+}\left(p_{l}\right)+v\left(p_{v}\right) .
\end{aligned}
$$

where $q=u, c$. The total squared matrix element $\left|\overline{\mathscr{M}}^{2}\right|$ of the process in Eq. (2.1) is defined as

$$
\left|\overline{\mathscr{M}}^{2}\right|=\sum_{L, R} \sum_{\left(\lambda_{t} \lambda_{t}^{\prime}= \pm\right)} \rho_{L R, \lambda_{t} \lambda_{t}^{\prime}}^{P^{t \bar{t}}} \rho_{\lambda_{t} \lambda_{t}^{\prime}}^{D^{t}}=\sum_{L, R\left(\lambda_{t} \lambda_{t}^{\prime}= \pm\right)} \mathscr{M}_{\lambda_{t}}^{L, R} \mathscr{M}_{\lambda_{t}^{\prime}}^{* L, R} \rho_{\lambda_{t} \lambda_{t}^{\prime}}^{D^{t}}
$$

where $\mathscr{M}_{\lambda_{t}}^{L, R}$ is the production helicity amplitude of the top with a given helicity $\lambda_{t}$ and the helicities of the anti-top is summed over. The production helicity amplitudes along with the decay matrix $\left(\rho_{\lambda_{t}}^{D_{t}^{t}}\right)$ of the top are explicitly given in the Appendix of Ref. [2]. The calculations are done in the frame with the electron beam in the positive $z$ direction, the top emitted at a polar angle $\theta_{t}$ and the quark emitted in the top decay making a polar angle $\theta_{q}$ with the electron beam.

The polar distribution of the emitted quark $q(=u, c)$, in the presence of beam polarization is

$$
\begin{aligned}
\frac{d \sigma}{d s d \cos \theta_{q} d \phi_{t}}= & \frac{1}{4}\left(\left(1-P_{e^{-}}^{L}\right)\left(1+P_{e^{+}}^{L}\right)\left|T_{e_{L}^{-} e_{R}^{+}}\right|^{2}+\left(1+P_{e^{-}}^{L}\right)\left(1-P_{e^{+}}^{L}\right)\left|T_{e_{R}^{-} e_{L}^{+}}\right|^{2}\right) \\
& -\frac{1}{2} P_{e^{-}}^{T} P_{e^{+}}^{T} \operatorname{Re} \mathrm{e}^{\mathrm{i}\left(\eta-2 \phi_{\mathrm{t}}\right)} \mathrm{T}_{\mathrm{e}_{\mathrm{R}}^{-} \mathrm{e}_{\mathrm{L}}^{+}} \mathrm{T}_{\mathrm{e}_{\mathrm{L}}^{-} \mathrm{e}_{\mathrm{R}}^{+}},
\end{aligned}
$$


where $\eta=\alpha_{-}+\alpha_{+}$, with $\alpha_{\mp}$ denoting the angle of polarization of the electron and the positron, respectively. The degree of the longitudinal and the transversal polarization for the electrons and positrons are denoted by $P_{e^{\mp}}^{L}$ and $P_{e^{\mp}}^{T}$, with $T_{e_{\lambda_{1}}^{-}} e_{\lambda_{2}}^{+}$being the helicity amplitude for the process under consideration. The baseline machine design for future colliders allows for up to $P_{e^{-}}^{L, T}=$ $\pm 80 \%$ electron (longitudinal and transversal) polarization, while provisions have been made to allow positron polarization of $P_{e^{+}}^{L, T}= \pm 30 \%$. The helicities of the electron and the positron are denoted by $\lambda_{1}, \lambda_{2}$ respectively and $\left|T_{e_{L}^{-} e_{R}^{+}}\right|^{2},\left|T_{e_{R}^{-} e_{L}^{+}}\right|^{2}, T_{e_{R}^{-} e_{L}^{+}}^{*} T_{e_{L}^{-} e_{R}^{+}}$are given by

$$
\begin{aligned}
\left|T_{e_{L}^{\mp} e_{R}^{ \pm}}\right|^{2}= & \left(\left|g_{t q}\right|^{2}+\left|g_{q t}\right|^{2}\right)\left(a_{0}+a_{1} \cos \theta_{q}+a_{2} \cos ^{2} \theta_{q}\right) \\
& \pm\left(\left|g_{t q}\right|^{2}-\left|g_{q t}\right|^{2}\right)\left(b_{0}+b_{1} \cos \theta_{q}+b_{2} \cos ^{2} \theta_{q}\right), \\
T_{e_{R}^{-} e_{L}^{+}}^{*} T_{e_{L}^{-} e_{R}^{+}}= & \left(3 \cos ^{2} \theta_{q}-1\right) \cos \left(\eta-2 \phi_{t}\right)\left\{\left(\left|g_{t q}\right|^{2}+\left|g_{q t}\right|^{2}\right) c_{0}+\left(\left|g_{t q}\right|^{2}-\left|g_{q t}\right|^{2}\right) d_{0}\right\},
\end{aligned}
$$

where $a_{i}, b_{i}, c_{0}, d_{0}$ coefficients are functions of the standard SM $\gamma$ and $Z$ couplings with the top and the leptons in the $t \bar{t}$ production. These equations are explicitly given in Ref. [2]. The Yukawa chiral couplings $\left|g_{t q}\right|^{2}$ and $\left|g_{q t}\right|^{2}$ are proportional to the polar angle of the emitted light quark, $\cos \theta_{q}$ and $\cos ^{2} \theta_{q}$, but the dependencies differ for $\left|g_{t q}\right|^{2}$ and $\left|g_{q t}\right|^{2}$. This makes possible to control the influence of the individual chiral couplings with a suitable choice of beam polarization.

\subsection{Angular Asymmetries at the ILC}

We consider here the polar angular distributions for different beam polarizations in the presence of chiral FCNC couplings and accordingly construct asymmetries. The results are presented considering $\sqrt{\left|g_{t q}\right|^{2}+\left|g_{q t}\right|^{2}}=0.16$, in accordance with the latest LHC bounds [1] and the SM background $\bar{t} W b$ is scaled down to be comparable with the signal. The initial results are presented without any cuts but a detailed analysis including the experimental cuts is also performed. The

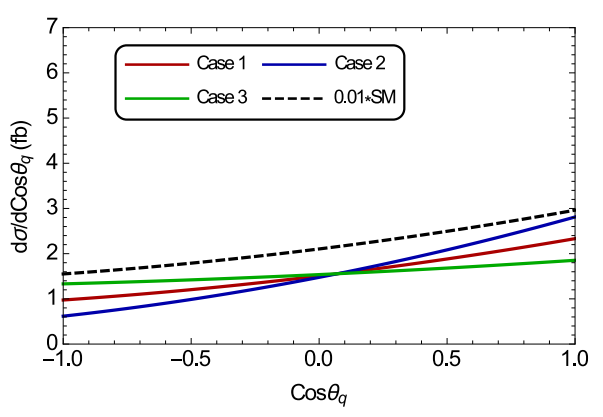

(a)

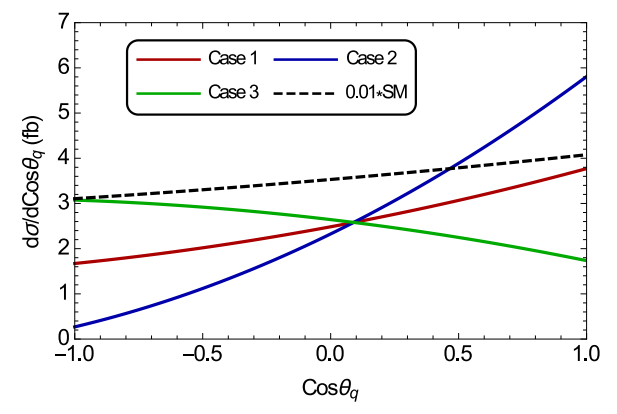

(b)

Figure 1: The polar angle distribution of the quark at $\sqrt{s}=500 \mathrm{GeV}$, for $(a) P_{e^{-}}^{L}=P_{e^{+}}^{L}=0$ and $(b)$ $P_{e^{-}}^{L}=-0.8, P_{e^{+}}^{L}=0.3$. The different Cases are discussed in the text.

polar angle distribution of the emitted quark, calculated from Eqs.(2.3, 2.4), is plotted in Fig. 1 for both the signal and the background. The polar angle distribution is sensitive to the chirality of the Yukawa couplings and the results are given for three different cases

- Case 1: $\sqrt{\left|g_{t q}\right|^{2}+\left|g_{q t}\right|^{2}}=0.16$, $\bullet$ Case $2: \sqrt{\left|g_{t q}\right|^{2}+\left|g_{q t}\right|^{2}}=0.16$, with $\left|g_{q t}\right|^{2}=0$

- Case 3: $\sqrt{\left|g_{t q}\right|^{2}+\left|g_{q t}\right|^{2}}=0.16$, with $\left|g_{t q}\right|^{2}=0$. 
The different Cases behave similar in the unpolarized case Fig. 1a, but for polarized beams all Cases clearly differ among each other, Fig. 1b. Therefore using the suitable initial beam polarization the dominance of one of the coupling if present will be clearly manifested. The azimuthal asymmetry displays a similar behaviour for the signal and the background, whereas the FB asymmetry distinguishes among the different cases and is sensitive to the beam polarization. We plot in Fig. 2 the FB asymmetry as a function of cut-off angle $\cos \theta_{0}$, with the dip signifying $A_{f b}\left(\cos \theta_{0}\right)=0$. We have

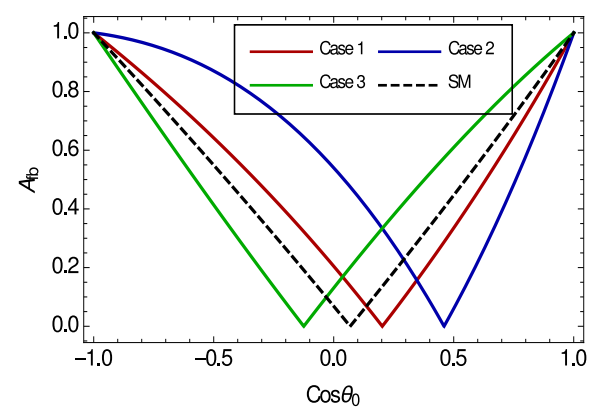

Figure 2: The forward backward asymmetry as a function of the cutoff angle $\cos \theta_{0}$ at $\sqrt{s}=500 \mathrm{GeV}$ for $P_{e^{-}}^{L}=-0.8, P_{e^{+}}^{L}=0.3$ See text for the different Cases.

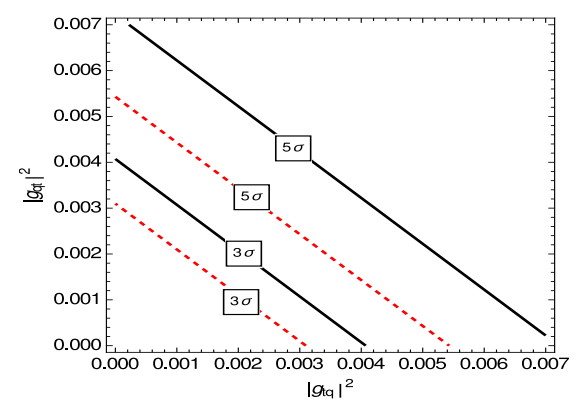

Figure 3: Contour plots in the $\left|g_{t q}\right|^{2}-$ $\left|g_{q t}\right|^{2}$ plane, for the statistical significance $S$, from the production c.s., with unpolarized beams [black] and $P_{e^{-}}^{L}=$ $-0.8, P_{e^{+}}^{L}=0.3$ [red-dashed].

also performed a full numerical study of the FCNC interactions in the $t \rightarrow q H$ decay at the ILC in the $t \bar{t}$-zero momentum frame (ZMF). We estimate the sensitivity which can be obtained for these FCNC couplings from the production cross section, by the efficient signal identification and the significant background suppression. In Fig. (3) the contours of $3 \sigma$ and $5 \sigma$ significance for our process in the $\left|g_{t q}\right|^{2}-\left|g_{q t}\right|^{2}$ plane are presented. The sensitivity of the linear collider will increase with the implementation of beam polarization with left polarized electrons and right polarized positrons.

\subsection{Top spin observables at the ILC}

The spin information of the decaying top is not diluted by hadronization. The spins of the top are correlated in top pair production, therefore the decay products of the top and the antitop are correlated. We consider the following spin observables

$$
\begin{aligned}
\mathscr{O}_{1} & =\frac{4}{3} \mathbf{S}_{t} \cdot \mathbf{S}_{\bar{t}}, & \mathscr{O}_{2} & =\mathbf{S}_{t} \cdot \hat{\mathbf{a}}, \quad \overline{\mathscr{O}}_{2}=\mathbf{S}_{\bar{t}} \cdot \hat{\mathbf{b}}, \\
\mathscr{O}_{3} & =4\left(\mathbf{S}_{t} \cdot \hat{\mathbf{a}}\right)\left(\mathbf{S}_{\bar{t}} \cdot \hat{\mathbf{b}}\right), & \mathscr{O}_{4} & =4\left(\left(\mathbf{S}_{t} \cdot \hat{\mathbf{p}}\right)\left(\mathbf{S}_{\bar{t}} \cdot \hat{\mathbf{q}}\right)+\left(\mathbf{S}_{t} \cdot \hat{\mathbf{q}}\right)\left(\mathbf{S}_{\bar{t}} \cdot \hat{\mathbf{p}}\right)\right),
\end{aligned}
$$

giving the net spin polarization of the top-antitop system $\left(\mathscr{O}_{1}\right)$, polarization of the top (antitop) quark $\left(\mathscr{O}_{2}\left(\overline{\mathscr{O}}_{2}\right)\right)$, the top-antitop spin correlation $\left(\mathscr{O}_{3}\right)$, with respect to spin quantization axes â and $\hat{\mathbf{b}}$. The observable $\mathscr{O}_{4}$ is an additional top-antitop spin correlation with respect to the momentum of the incoming and the outgoing particles [3]. The observable $\mathscr{O}_{1}$ can be probed using the opening angle distribution $(\varphi)$, i.e. the angle between the direction of flight of the two (top and antitop) spin analysers (final particles produced in the top and antitop decays), defined in the $t$ and $\bar{t}$ frames, 
respectively, i.e $\hat{\mathbf{p}}_{q} \cdot \hat{\mathbf{p}}_{l}=\cos \varphi$.

$$
\frac{1}{\sigma} \frac{d \sigma}{d \cos \varphi}=\frac{1}{2}(1-D \cos \varphi), \quad D=\left\langle\mathscr{O}_{1}\right\rangle \kappa_{q} \kappa_{l^{-}}
$$

The spin analyzer for the FCNC top-Higgs decays can be any of the decay product of the top (antitop). We consider the $c / u$ quark from the top and the $l^{-}$from the anti-top as spin analysers in this work. The spin analysing power of the quark from $t \rightarrow q H,(q=u, c)$ and $l$ from the antitop decay is given by $\kappa_{q}=\left(\left|g_{q t}\right|^{2}-\left|g_{t q}\right|^{2}\right) /\left(\left|g_{q t}\right|^{2}+\left|g_{t q}\right|^{2}\right), \quad \kappa_{l}=1$.

The leptons due to the $V-A$ interactions are the perfect spin analysers, with their flight directions $100 \%$ correlated with the directions of the antitop spin. It is clear from above that with $\left|g_{q t}\right|^{2} \simeq\left|g_{t q}\right|^{2}$, the spin information of the top will be lost $\left(\kappa_{q} \approx 0\right)$. However in the presence or dominance of only one of the coupling, the emitted quark acts as a perfect spin analyser $\left(\kappa_{q} \approx 1\right)$. The observables $\left(\mathscr{O}_{2}, \overline{\mathscr{O}}_{2}, \mathscr{O}_{3}\right)$ can be measured using the double differential angular distribution of the top and antitop quark decay products:

$$
\frac{1}{\sigma} \frac{d^{2} \sigma}{d \cos \theta_{f} d \cos \theta_{\bar{f}}}=\frac{1}{4}\left(1+B_{t} \cos \theta_{f}+B_{\bar{t}} \cos \theta_{\bar{f}}-C \cos \theta_{f} \cos \theta_{\bar{f}}\right),
$$

where $\theta_{f}\left(\theta_{\bar{f}}\right)$ is the angle between the direction of the top (antitop) spin analyser $f,(\bar{f})$ in the $t(\bar{t})$ rest frame. Comparing Eq. (2.7) with Eq. (2.5) we have

$$
B_{t}=\left\langle\mathscr{O}_{2}\right\rangle \kappa_{q}, \quad B_{\bar{t}}=\left\langle\overline{\mathscr{O}}_{2}\right\rangle \kappa_{l^{-}}, \quad C=\left\langle\mathscr{O}_{3}\right\rangle \kappa_{q} \kappa_{l^{-}} .
$$

The arbitrary unit vectors $\hat{\mathbf{a}}$ and $\hat{\mathbf{b}}$ in Eq. (2.5) specify different spin quantization axes which can be chosen to maximize the desired polarization and the correlation effects, and we take them as

$$
\begin{array}{ll}
\hat{\mathbf{a}}=-\hat{\mathbf{b}}=\hat{\mathbf{q}}, & \text { ("helicity" basis) }, \\
\hat{\mathbf{a}}=\hat{\mathbf{b}}=\hat{\mathbf{p}}, & \text { ("beamline" basis), } \\
\hat{\mathbf{a}}=\hat{\mathbf{b}}=\hat{\mathbf{d}}_{\mathrm{SM}}=\hat{\mathbf{d}}_{\mathrm{SM}}^{\max }=\frac{-\hat{\mathbf{p}}+(1-\gamma) z \hat{\mathbf{q}}_{1}}{\sqrt{1-\left(1-\gamma^{2}\right) z^{2}}}, & \text { ("off - diagonal" basis), }
\end{array}
$$

where $\hat{\mathbf{q}}=\hat{\mathbf{q}}_{1}$ is the direction of the outgoing top quark and $\hat{\mathbf{p}}$ is the direction of the incoming beam, both in the $t \bar{t}$ center of mass frame. The off-diagonal basis [4] is the one, where the top spins are $100 \%$ correlated $\left(z=\hat{\mathbf{p}} \cdot \hat{\mathbf{q}}_{1}=\cos \theta\right.$ and $\left.\gamma=E_{t} / m_{t}=1 / \sqrt{1-\beta^{2}}\right)$ and interpolates between the beamline basis at the threshold $(\gamma \rightarrow 1)$ and the helicity basis for ultrarelativistic energies $(\gamma \rightarrow$ $\infty)[4,5]$. In Table 1 we present the values of the different spin observables in the different spin basis considered here, in the presence of beam polarizations. We note that the top (antitop) spin polarizations are quite sensitive to the beam polarization, with all observables being proportional to $\kappa_{f}=\kappa_{q}$ and will be equal to zero if $g_{t q}$ and $g_{q t}$ are equal. The chiral nature of the FCNC coupling is more clearly visible in the 'beamline' and the 'off-diagonal' basis, with the beam polarizations of $P_{e^{-}}^{L}=-0.8$ and $P_{e^{+}}^{L}=0.3$. The same happens also for spin-spin correlations [2].

\section{Conclusion}

We have explored the chiral structure of the flavor-violating top-Higgs interactions through the $t \bar{t}$ production at polarized linear colliders. The LHC is currently not sensitive to the chirality of these couplings as both the branching ratio of the top to $q H$ and the total production cross 


\begin{tabular}{|c|c|c|c|c|}
\hline Observables & Basis & $P_{e^{-}}^{L}=0, P_{e^{+}}^{L}=0$ & $P_{e^{-}}^{L}=0.8, P_{e^{+}}^{L}=-0.3$ & $P_{e^{-}}^{L}=-0.8, P_{e^{+}}^{L}=0.3$ \\
\hline $\mathscr{O}_{1}$ & & $0.333 \kappa_{f}$ & $0.333 \kappa_{f}$ & $0.333 \kappa_{f}$ \\
\hline & hel & $-0.076 \kappa_{f}$ & $0.247 \kappa_{f}$ & $-0.239 \kappa_{f}$ \\
& beam & $-0.174 \kappa_{f}$ & $0.344 \kappa_{f}$ & $-0.436 \kappa_{f}$ \\
$\mathscr{O}_{2}$ & off & $0.176 \kappa_{f}$ & $-0.351 \kappa_{f}$ & $0.443 \kappa_{f}$ \\
& min & $0.04 \kappa_{f}$ & $-0.131 \kappa_{f}$ & $0.127 \kappa_{f}$ \\
\hline & hel & $-0.654 \kappa_{f}$ & $-0.666 \kappa_{f}$ & $-0.648 \kappa_{f}$ \\
& beam & $0.881 \kappa_{f}$ & $0.852 \kappa_{f}$ & $0.897 \kappa_{f}$ \\
$\mathscr{O}_{3}$ & off & $0.911 \kappa_{f}$ & $0.886 \kappa_{f}$ & $0.924 \kappa_{f}$ \\
& min & $0.224 \kappa_{f}$ & $0.229 \kappa_{f}$ & $0.222 \kappa_{f}$ \\
\hline $\mathscr{O}_{4}$ & & $0.546 \kappa_{f}$ & $0.612 \kappa_{f}$ & $0.512 \kappa_{f}$ \\
\hline
\end{tabular}

Table 1: The value of the spin observables in different bases, with different choices of initial beam polarization. $\kappa_{f}=\kappa_{q}$ for FCNC $t$-decays and $\kappa_{f}=\kappa_{b}$ for $t \rightarrow W^{+} b$.

section measured at the LHC are proportional to $\left|g_{t q}\right|^{2}+\left|g_{q t}\right|^{2}$. We have considered various observables such as the polar angle distribution of the quark emitted from the $t \rightarrow q H$, forward-backward asymmetry along with the top spin observables, which exhibit a behaviour sensitive to the nature of the coupling. The sensitivity of the various observables on the FCNC parameters can be enhanced with a suitable choice of initial beam polarization. We show in Fig. 4 the $3 \sigma$ and the

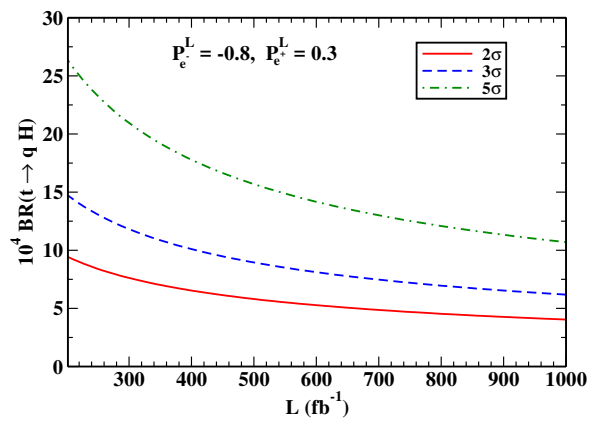

Figure 4: The sensitivity of $3 \sigma$ and $5 \sigma$ to $\mathrm{BR}(t \rightarrow q H)$ at $\sqrt{s}=500 \mathrm{GeV}$, as a function of the luminosity, $\mathscr{L}$.

$5 \sigma$ upper bounds on the branching ratios, which can be obtained at the ILC, at $\sqrt{s}=500 \mathrm{GeV}$, with the choice of beam polarization $P_{e^{-}}^{L}=-0.8, P_{e^{+}}^{L}=0.3$. The $\mathrm{BR}(t \rightarrow q H)$ can be probed to $5.59 \times 10^{-3}\left(8.84 \times 10^{-4}\right)$ at $3 \sigma$ level at the ILC, with $\sqrt{s}=500 \mathrm{GeV}, \mathscr{L}=500 \mathrm{fb}^{-1}$ and a beam polarization of $P_{e^{-}}^{L}=0(-0.8), P_{e^{+}}^{L}=0(0.3)$.

\section{References}

[1] Greljo A, Kamenik J F and Kopp J, JHEP 1407, 046 (2014) (Preprint arXiv:1404.1278 [hep-ph])

[2] Melić B and Patra M, JHEP 1701, 048 (2017) (Preprint arXiv:1610.02983 [hep-ph])

[3] Brandenburg A, Flesch M and Uwer P, Phys. Rev. D 59, 014001 (1999) (Preprint hep-ph/9806306)

[4] Parke S J and Shadmi Y, Phys. Lett. B 387, 199 (1996) (Preprint hep-ph/9606419)

[5] Fajfer S, Kamenik J F and Melić B, JHEP 1208 (2012) 114 (Preprint arXiv:1205.0264 [hep-ph]) 